\title{
Investigation of Single Nucleotide Polymorphisms in the Adipocyte Fatty-Acid Binding Protein (FABP4) Gene
}

\author{
Sang Wook Kim, Ji Hye Jung, Kwan Suk Kim ${ }^{1}$, Cheol Koo Lee ${ }^{2}$, Jong Joo Kim ${ }^{3}$, Bong Hwan Choi ${ }^{4}$, \\ Tae Hun $\mathrm{Kim}^{4}, \mathrm{Ki}$ Duk Song ${ }^{5}$ and Byung Wook Cho* \\ Department of Animal Science, Pusan National University, Kyung Nam, Miryang 627-706, Korea \\ ${ }^{1}$ Department of Animal Science, Chungbuk National University, Cheongju 361-763, Korea \\ ${ }^{2}$ College of Life Sciences \& Biotechnology, Korea University, Seoul 136-701, Korea \\ ${ }^{3}$ Department of Biotechnology, Yeungnam National University, Gyeongsan 712-749, Korea \\ ${ }^{4}$ Animal Genomics and Bioinformatics Division, National Livestock Research Institute, RDA, Stwon 441-707, Korea \\ ${ }^{5}$ NICHD/National Institutes of Health, 9000 Rockville Pike, Bethesda, MD 20892, USA
}

Received October 22, 2007 / Accepted November 10, 2007

\begin{abstract}
We found 8 single nucleotide polymorphisms (SNPs) in adipocyte fatty acid bonding protein (FABP4) gene as candidate gene of FAT1 locus on pig chromosome 4 . With over 800 heads of major commercial pig breeds including Duroc, Landrace, Berkshire and Yorkshire, we analyzed SNPs of FABP4 gene to determine possible effects of FABP4 genotype to economically important traits. $400 \sim 800 \mathrm{bp}$ amplicons in FABP4 gene were used PCR-RFLP for each SNPs and we found that the frequency of some SNPs of this gene was different among the breeds. According to the statistical analyses to determine possible associations of each genotype with economic traits, it was found that subgroup with different genotypes showed significant differences in daily gain, backfat thickness, lean percentage and feed conversion ratio $(\mathrm{P}<0.05)$. Thus, as a part of enhancing the selection competence related to swine growth rate and lean percentage, it is expected that FABP4 gene markers verified in this study will be useful to use for Korean commercial pig industry.
\end{abstract}

Key words : Economic traits, swine, SNP Detection, PCR-RFLP, polymorphism, FABP

\section{Introduction}

FABP4 gene is located on pig chromosome 4 close to FAT1 loci which is considered one of the most important quantitative trait loci (QTL) and was reported as QTL from domestic animals for the first time. It has been reported that FABP4 has critical effects on obesity and growth [2]. Although many studies confirmed the FAT1 QTL on pig chromosome 4 from resource populations generated for QTL studies, causative gene (s) that might be responsible for the FAT1 QTL remains to be identified $[3,7,9]$. This situation contrasts well with other cloned loci like $\mathrm{RN}$ or IGF2. It is of interest why FAT1 has been not yet identified, but one of possible reasons is that more than 2 loci included in FAT1 make it more challenging to search for any causative mutation [10].

This hypothesis was supported with statistical QTL analysis including FABP4 gene in crossbred herds of

\footnotetext{
*Corresponding author

Tel : +82-55-350-5515, Fax : +82-55-350-5519

E-mail : bwcho@pusan.ac.kr
}

Landrace and Iberian (IBMAP).

Some studies reported that FABP4 is a major player for oxidation or esterification in plasma membrane to facilitate fatty acid to be conveyed.

According to conventional literatures, micorsatellite analysis revealed that microsatellite within the first intron of FABP4 gene in Duroc is associated with fat content within muscle [4]. Another study reported that FABP4 is a candidate gene for lipid metabolism, so this study sought to analyze FABP4 polymorphisms in IBMAP crossing hybrids [8].

Genotypes of the most beneficial polymorphism (e.g. SNP in the 3rd intron, insertion/ deletion in the 1st intron, etc) were characterized in Ibreian $X$ Landrace cross hybrids. After analyzing QTL, single marker and haplotype, more than two quantitative trait genes were located in FAT1 region, and FABP4 polymorphisms were closely associated with obesity [1].

According to comparison of alleles from 4 breeds used herein, it was found that Del2634C polymorphisms occurred under indirect selection. In addition, it was also 
found that FABP4 is closely associated with obesity, not growth [6]. As indicated from previous studies [5], the FABP4 might be one of major genes in pigs controlling fat deposition. Here, we aimed to analyze the differences in the frequency of FABP4 mutations among commercial breeds, performed the association study to determine the possible effect of these SNPs of FABP4 gene on each trait. Thus, this study examined possible associations between economic traits of each swine breed (e.g. backfat thickness, daily gain, feed conversion ratio, and lean percentage) and thereby explored the feasibility of FABP4 gene in screening superior boars on the field of swine breeding industry.

\section{Materials and Methods}

\section{Animal Material and Traits Analyzed}

Experimental animals which consisted of Duroc, Landrace, Berkshire and Yorkshire were admitted and bred in the laboratory of Korea Swine Association (KSA) from January 2006 to January 2007. Phenotypic data of these animals which included daily gain, feed conversion ratio, backfat thickness and lean percentage, were measured and collected until body weight reached to $90 \mathrm{~kg}$ from $30 \mathrm{~kg}$ (Table Genomic DNAs were extracted from the bleeds of experimental animals using Genomic DNA Extraction Kit (Promega) and Toyobo MagExtractor Kit (Toyobo).

\section{Amplification and Sequencing of Swine FABP4 Gene}

The sequence information of Landrace registered in National Center for Biotechnology Information (NCBI) was used (total $6300 \mathrm{bp}$ ) across 5'UTR to 3'UTR region, covering 5 exons and 5 introns (Fig 1). For PCR amplification, total 10 primers were designed using Oligo 6 sofrware to generate $400 \sim 800$ bp PCR products (Table 2).

Before PCR amplification, commercial breeds DNAs were amplified from the pooling DNA panel of 4 swine breeds (Duroc, Landrace, Yorkshire and Berkshire) by means of hot start PCR Taq polymerase. In terms of PCR products, each DNA panel's sequences could be obtained through $\mathrm{ABI} 3700$ sequencer. Those sequences were analyzed using Sequencer 4.6 software to find out SNPs as index of differences in traits among breeds. To demonstrate restriction fragment length polymorphism (RFLP) of each SNPs, restriction enzymes were chosen using NEB cutter (http://tools.neb.com/NEBcutter2/index.php) (Table 3). PCR-RFLP test was conducted using DNA samples from total 800 swine bleeds (200 per breed) according to finding of total 8 single nucleotide mutations (Table 4).

\section{Statistical Analyses}

In order to estimate the effects of FABP4 SNP genotypes on economic traits of commercial breeds studied, General

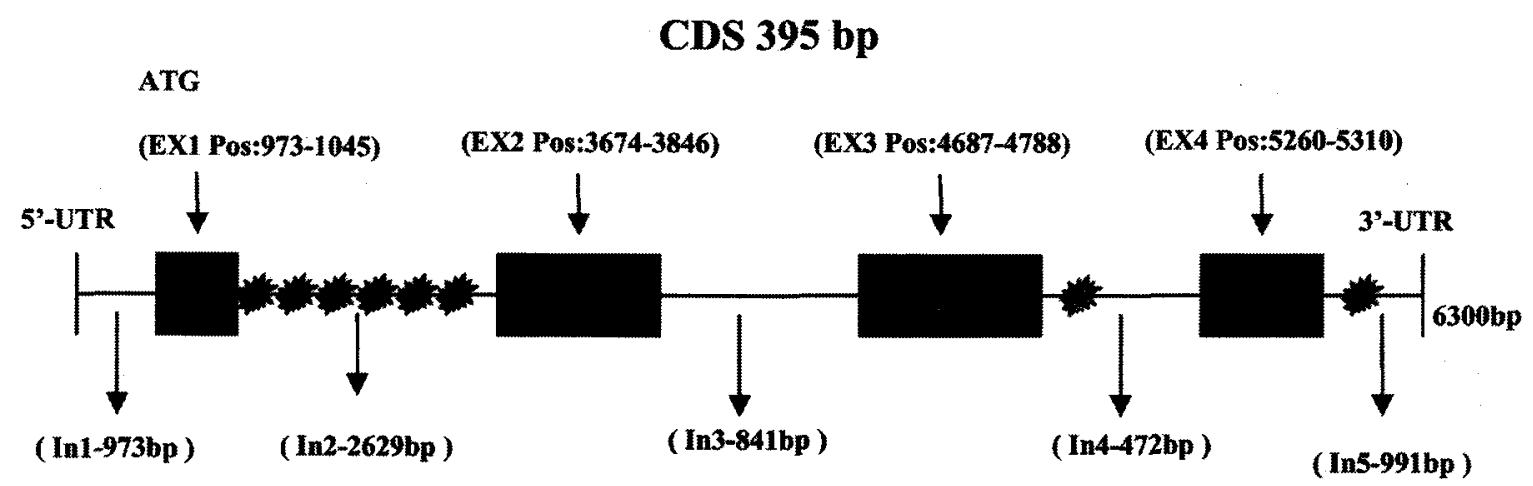

Fig. 1. Gene map and polymorphism in FABP4 on porcine chromosome 4.

(Coding exon are marked by black block) - SNP SITE.

Table 1. Summary of overall means and standard deviations on performance traits

\begin{tabular}{ccccc}
\hline Breed & Daily Gain $(\mathrm{g})$ & Feed Conversion $(\%)$ & Backfat $(\mathrm{cm})$ & Meat Percentage $(\%)$ \\
\hline $\mathrm{DD}(\mathrm{n}=200)$ & $1116.78 \pm 79.32$ & $2.26 \pm 0.14$ & $1.39 \pm 0.20$ & $58.20 \pm 2.43$ \\
$\mathrm{LL}(\mathrm{n}=200)$ & $1020.10 \pm 92.89$ & $2.34 \pm 0.14$ & $1.40 \pm 0.26$ & $55.53 \pm 2.99$ \\
$\mathrm{YY}(\mathrm{n}=200)$ & $1026.33 \pm 92.57$ & $2.33 \pm 0.12$ & $1.29 \pm 0.16$ & $56.29 \pm 2.19$ \\
$\mathrm{BB}(\mathrm{n}=200)$ & $1044.91 \pm 96.90$ & $2.29 \pm 0.14$ & $1.37 \pm 0.21$ & $57.25 \pm 2.89$ \\
\hline
\end{tabular}

DD: Duroc, LL: Landrace, YY: Yorkshire, BB: Berkshire 
Table 2. PCR primers and conditions used for amplification and sequencing

\begin{tabular}{|c|c|c|c|c|}
\hline \multirow{2}{*}{ Amplicon } & \multicolumn{2}{|c|}{ Primer } & \multirow{2}{*}{$\begin{array}{l}\text { Annealing } \\
\text { Temp. }\end{array}$} & \multirow{2}{*}{$\begin{array}{l}\text { product } \\
\text { size }\end{array}$} \\
\hline & Forward $\left(5^{\prime} \rightarrow 3^{\prime}\right)$ & Reverse $\left(5^{\prime} \rightarrow 3^{\prime}\right)$ & & \\
\hline Fabp4-1 & TTA GAT TTT GAT GGC TTG TGA & CGG GTC ATT GGA CTT AGA G & 56 & 644 \\
\hline Fabp4-2 & GCC GAA CAC TAA ATG GTC AGA & GCA AAG AGC CAA GTA AGC CAA & 60 & 700 \\
\hline Fabp4-3 & TCT TAA AGG TGA ACT GAT ATG & CTT GGT CTC ATT CAT GC & 58 & 726 \\
\hline Fabp4-4 & TAT AGG CAA GAG GGT AAT CA & ATG GGA TGA ATA ATT TAC AGC & 56 & 718 \\
\hline Fabp4-5 & TGT GAT GGG CTG TAA AT & GCA GGT AAA TAA AGT AGG CTA & 55 & 727 \\
\hline Fabp4-6 & TGT TCC AGG AGA ATC TTT GT & CCT TAT TCC TCA CCT TGA CTT & 60 & 697 \\
\hline Fabp4-7 & CCT GGT TCC TAA AAG ACT GTT & TTT CTG TTT ATG GTG GTT GTC & 63 & 885 \\
\hline Fabp4-8 & GCA GAA GTG GGA TGG A & TAT TGA ACA AAA TCA GTC TGG & 55 & 713 \\
\hline Fabp4-9 & GAG AGC ATA AGC CAA ATT GTA & GCT GAG GCT GTG GTA TAG G & 58 & 534 \\
\hline Fabp $4-10$ & CAC AGC CTC AGC ACC TC & TTT GGA AAC CAC TAC AAG AAC & 53 & 446 \\
\hline
\end{tabular}

Table 3. The number of SNPs, polymorphic sequence and restriction enzyme for RFLP in pigs from Korea commercial line

\begin{tabular}{|c|c|c|c|c|}
\hline Gene & No. of SNP & Polymorphic sequence & Different base in each breed & Restriction Enzyme for RFLP \\
\hline \multirow{8}{*}{ FABP4 } & \multirow{8}{*}{8} & $\mathrm{D} / \mathrm{Y} / \mathrm{B}: \mathrm{TCA}(\mathrm{A} / \mathrm{G}) \mathrm{TGA}$ & $\mathrm{D}, \mathrm{Y}, \mathrm{B}: \mathrm{R} / \mathrm{L}: \mathrm{A}$ & $\mathrm{BapHI}$ \\
\hline & & D/L: TTT(T/G)AAC & $\mathrm{D}, \mathrm{L}: \mathrm{K} / \mathrm{Y}: \mathrm{T} / \mathrm{B}: \mathrm{T}$ & Not available \\
\hline & & D/Y: TTA(A/G)TAT & $\mathrm{D} / \mathrm{Y}: \mathrm{R} / \mathrm{L}: \mathrm{A} / \mathrm{B}: \mathrm{A}$ & Not available \\
\hline & & $\mathrm{D} / \mathrm{Y}: \mathrm{GCA}(\mathrm{A} / \mathrm{G}) \mathrm{CTT}$ & $\mathrm{D} / \mathrm{Y}: \mathrm{R} / \mathrm{L}: \mathrm{A} / \mathrm{B}: \mathrm{A}$ & Fnu4HI \\
\hline & & $\mathrm{D} / \mathrm{L} / \mathrm{Y}: \mathrm{AGT}(\mathrm{T} / \mathrm{C}) \mathrm{GAT}$ & $D / L / Y: Y / B: T$ & MsI I \\
\hline & & $\mathrm{D} / \mathrm{Y} / \mathrm{B}: \mathrm{AGT}(\mathrm{T} / \mathrm{C}) \mathrm{GGC}$ & $\mathrm{D} / \mathrm{Y} / \mathrm{B}: \mathrm{Y} / \mathrm{L}: \mathrm{T}$ & Fnu4HI \\
\hline & & D/L: TGC(C/A)TAG & $\mathrm{D} / \mathrm{L}: \mathrm{M} / \mathrm{Y}: \mathrm{C} / \mathrm{B}: \mathrm{C}$ & Bfa I \\
\hline & & D/L: ACA(A/G)AAG & $\mathrm{D} / \mathrm{L}: \mathrm{R} / \mathrm{Y}: \mathrm{A} / \mathrm{B}: \mathrm{A}$ & Not available \\
\hline
\end{tabular}

DD: Duroc, LL: Landrace, YY: Yorkshire, BB: Berkshire

Table 4. FABP4 genotypes in four commercial pig breeds

\begin{tabular}{|c|c|c|c|c|c|c|c|c|c|c|c|c|c|c|c|}
\hline \multirow{2}{*}{ Breed } & \multicolumn{3}{|c|}{$\mathrm{A} 1761 \mathrm{G}(\mathrm{n}=200)$} & \multicolumn{3}{|c|}{ A2521G $(n=200)$} & \multicolumn{3}{|c|}{ T2713C $(n=200)$} & \multicolumn{3}{|c|}{ T3182C $(n=200)$} & \multicolumn{3}{|c|}{ C4858A $(n=200)$} \\
\hline & $\mathrm{AA}$ & $\mathrm{AG}$ & GG & $\mathrm{AA}$ & AG & GG & $\mathrm{TT}$ & $\mathrm{TC}$ & $\mathrm{CC}$ & TT & $\mathrm{TC}$ & $\mathrm{CC}$ & $\mathrm{CC}$ & $\mathrm{CA}$ & AA \\
\hline$D D$ & 5 & 152 & 43 & 25 & 85 & 90 & 11 & 169 & 20 & 6 & 111 & 73 & 2 & 160 & 38 \\
\hline LL & 69 & 35 & 46 & 85 & 57 & 58 & 12 & 124 & 64 & 154 & 39 & 7 & 101 & 59 & 40 \\
\hline$Y Y$ & 90 & 102 & 3 & 20 & 50 & 130 & 32 & 96 & 72 & 9 & 161 & 30 & 70 & 53 & 70 \\
\hline$B B$ & 62 & 67 & 71 & 76 & 60 & 64 & 102 & 40 & 58 & 20 & 146 & 34 & 5 & 65 & 130 \\
\hline
\end{tabular}

DD: Duroc, LL: Landrace, YY: Yorkshire, BB: Berkshire

Linear Model (GLM) analysis was perforrmed using SAS 9.1 Package/PC with parameters of breeds and FABP4 genotypes. To determine possible effects of FABP4 genotypes on each trait, we conducted significance test between least square means (Table 5).

\section{Results and Discussion}

\section{Analysis on the Frequency of FABP4 Genotypes in Each Breed}

The frequency of FABP4 genotypes in major four commercial breeds admitted and bred in KSA test status is presented in Table 4. It was found that SNPs of each breed (Duroc, Landrace, Yorkshire and Berkshire) were highly polymorphic in each trait. The effects of each SNP on traits of each swine breed were illustrated in Table 5. And FABP4 (A2521G) both Duroc and Yorkshire group, which showed higher growth rate than other 2 breeds, had higher frequency of FABP4 genotypes, and also showed higher significances in backfat thickness and lean percentage as 2 traits in potential association with growth rate than other 2 breeds.

\section{Analysis on Associations in Traits of FABP4 Genotypes}

Table 5 shows possible effects of FABP4 genotypes on each trait. In Duroc group, it was found that there were significant differences in backfat thickness and lean percentage 
Table 5. Least squares means and standard errors for the performance traits by FABP4 genotypes in four breeds combined data ( $\mathrm{n}=800)$

\begin{tabular}{|c|c|c|c|c|c|c|}
\hline $\begin{array}{l}\text { Region of } \\
\text { SNP }\end{array}$ & Breed & $\begin{array}{c}\text { FABP4 } \\
\text { genotype }\end{array}$ & Daily Gain(g) & Feed Conversion(\%) & Backfat $(\mathrm{cm})$ & Meat Percentage $(\%)$ \\
\hline \multirow{4}{*}{$\mathrm{A} 1761 \mathrm{G}$} & DD & $\begin{array}{l}\text { AA } \\
\text { AG } \\
\text { GG }\end{array}$ & $\begin{array}{l}1192.333 \pm 39.289 \\
1104.753 \pm 11.585 \\
1104.625 \pm 17.012\end{array}$ & $\begin{array}{l}2.161 \pm 0.062 \\
2.278 \pm 0.018 \\
2.298 \pm 0.027\end{array}$ & $\begin{array}{l}1.330 \pm 0.058 \\
1.304 \pm 0.017 \\
1.381 \pm 0.025\end{array}$ & $\begin{array}{l}58.500 \pm 1.085 \\
a_{58.707 \pm 0.320} \\
b_{57.321 \pm 0.470}\end{array}$ \\
\hline & LL & $\begin{array}{l}\text { AA } \\
\text { AG } \\
\text { GG }\end{array}$ & $\begin{array}{r}1013.125 \pm 16.643 \\
989.115 \pm 18.463 \\
960.461 \pm 26.111\end{array}$ & $\begin{array}{l}2.367 \pm 0.023 \\
2.392 \pm 0.026 \\
2.382 \pm 0.037\end{array}$ & $\begin{array}{l}1.466 \pm 0.056 \\
1.589 \pm 0.062 \\
1.541 \pm 0.088\end{array}$ & $\begin{array}{l}56.656 \pm 0.697 \\
55.319 \pm 0.774 \\
56.138 \pm 1.094\end{array}$ \\
\hline & $\mathrm{YY}$ & $\begin{array}{l}\mathrm{AA} \\
\mathrm{AG} \\
\mathrm{GG}\end{array}$ & $\begin{array}{r}a 963.411 \pm 25.749 \\
\mathrm{~b}^{\mathrm{b}} 1040.204 \pm 16.005 \\
\mathrm{~b}_{\mathrm{b}} 1035.000 \pm 22.634\end{array}$ & $\begin{array}{l}2.441 \pm 0.029 \\
2.368 \pm 0.018 \\
2.369 \pm 0.025\end{array}$ & $\begin{array}{l}1.398 \pm 0.054 \\
1.384 \pm 0.033 \\
1.320 \pm 0.054\end{array}$ & $\begin{array}{l}58.076 \pm 0.756 \\
57.279 \pm 0.470 \\
57.677 \pm 0.664\end{array}$ \\
\hline & $\mathrm{BB}$ & $\begin{array}{l}\text { AA } \\
\text { AG } \\
\text { GG }\end{array}$ & $\begin{array}{l}1003.523 \pm 58.123 \\
1006.666 \pm 57.783 \\
1009.941 \pm 24.274\end{array}$ & $\begin{array}{l}2.289 \pm 0.109 \\
2.280 \pm 0.099 \\
2.295 \pm 0.041 \\
\end{array}$ & $\begin{array}{l}1.301 \pm 0.056 \\
1.343 \pm 0.107 \\
1.247 \pm 0.045 \\
\end{array}$ & $\begin{array}{l}56.545 \pm 0.982 \\
58.000 \pm 1.493 \\
57.417 \pm 0.627 \\
\end{array}$ \\
\hline \multirow{4}{*}{ A2521G } & $\mathrm{DD}$ & $\begin{array}{l}\mathrm{AA} \\
\mathrm{AG} \\
\mathrm{GG}\end{array}$ & $\begin{array}{l}1066.166 \pm 14.131 \\
{ }^{*} 1140.981 \pm 12.349 \\
{ }^{*} 1119.700 \pm 28.961\end{array}$ & $\begin{array}{l}2.348 \pm 0.022 \\
2.227 \pm 0.019 \\
{ }^{*} a_{2.262 \pm 0.045}\end{array}$ & $\begin{array}{l}1.344 \pm 0.022 \\
1.326 \pm 0.019 \\
1.278 \pm 0.046\end{array}$ & $\begin{array}{l}58.109 \pm 1.421 \\
58.343 \pm 0.368 \\
58.660 \pm 0.863\end{array}$ \\
\hline & LL & $\begin{array}{l}\mathrm{AA} \\
\mathrm{AG} \\
\mathrm{GG}\end{array}$ & $\begin{array}{l}997.553 \pm 12.835 \\
985.153 \pm 26.639 \\
976.500 \pm 67.917\end{array}$ & $\begin{array}{l}2.376 \pm 0.018 \\
2.386 \pm 0.037 \\
2.420 \pm 0.096\end{array}$ & $\begin{array}{l}1.543 \pm 0.043 \\
1.457 \pm 0.089 \\
1.450 \pm 0.228\end{array}$ & $\begin{array}{l}55.970 \pm 0.531 \\
56.607 \pm 1.103 \\
57.200 \pm 2.814\end{array}$ \\
\hline & $\mathrm{YY}$ & $\begin{array}{l}\mathrm{AA} \\
\mathrm{AG} \\
\mathrm{GG}\end{array}$ & $\begin{array}{r}{ }^{a} 1033.716 \pm 13.782 \\
b_{1} 1011.950 \pm 23.872 \\
{ }^{a} 885.000 \pm 61.637\end{array}$ & $\begin{array}{l}2.370 \pm 0.015 \\
2.407 \pm 0.027 \\
2.493 \pm 0.069\end{array}$ & $\begin{array}{l}1.354 \pm 0.028 \\
1.397 \pm 0.050 \\
1.503 \pm 0.129\end{array}$ & $\begin{array}{l}57.628 \pm 0.402 \\
57.555 \pm 0.697 \\
55.900 \pm 1.800\end{array}$ \\
\hline & $B B$ & $\begin{array}{l}\mathrm{AA} \\
\mathrm{AG} \\
\mathrm{GG}\end{array}$ & $\begin{array}{l}1009.312 \pm 25.022 \\
1010.000 \pm 50.045 \\
1008.941 \pm 30.474\end{array}$ & $\begin{array}{l}2.301 \pm 0.042 \\
2.260 \pm 0.085 \\
2.200 \pm 0.065 \\
\end{array}$ & $\begin{array}{l}1.284 \pm 0.045 \\
1.172 \pm 0.097 \\
1.257 \pm 0.033 \\
\end{array}$ & $\begin{array}{l}57.256 \pm 0.635 \\
58.500 \pm 1.271 \\
56.917 \pm 0.891 \\
\end{array}$ \\
\hline \multirow{4}{*}{$\mathrm{T} 2713 \mathrm{C}$} & DD & $\begin{array}{l}\text { TT } \\
\text { TC } \\
\text { CC }\end{array}$ & $\begin{array}{l}1080.111 \pm 22.971 \\
1115.626 \pm 11.253 \\
1115.111 \pm 26.047\end{array}$ & $\begin{array}{l}2.325 \pm 0.036 \\
2.271 \pm 0.017 \\
2.251 \pm 0.041\end{array}$ & $\begin{array}{l}1.305 \pm 0.034 \\
1.337 \pm 0.016 \\
1.311 \pm 0.039\end{array}$ & $\begin{array}{l}58.072 \pm 0.643 \\
58.250 \pm 0.315 \\
58.072 \pm 0.729\end{array}$ \\
\hline & LL & $\begin{array}{l}\text { TT } \\
\text { TC } \\
\text { CC }\end{array}$ & $\begin{array}{r}965.086 \pm 19.467 \\
1013.476 \pm 14.406 \\
976.666 \pm 38.114\end{array}$ & $\begin{array}{l}a_{2} .440 \pm 0.026 \\
b_{2.345 \pm 0.019} \\
b_{2.380 \pm 0.052}\end{array}$ & $\begin{array}{r}1.654 \pm 0.064 \\
b 1.485 \pm 0.047 \\
1.303 \pm 0.125\end{array}$ & $\begin{array}{l}54.826 \pm 0.805 \\
56.442 \pm 0.596 \\
58.250 \pm 1.577\end{array}$ \\
\hline & $Y Y$ & $\begin{array}{l}\text { TT } \\
\text { TC } \\
\text { CC }\end{array}$ & $\begin{array}{r}1040.555 \pm 14.613 \\
1000.272 \pm 22.895 \\
960.142 \pm 40.589\end{array}$ & $\begin{array}{l}2.375 \pm 0.016 \\
2.393 \pm 0.026 \\
2.415 \pm 0.046\end{array}$ & $\begin{array}{l}1.347 \pm 0.030 \\
1.432 \pm 0.047 \\
1.348 \pm 0.084\end{array}$ & $\begin{array}{l}57.900 \pm 0.420 \\
56.677 \pm 0.658 \\
57.571 \pm 1.167\end{array}$ \\
\hline & $\mathrm{BB}$ & $\begin{array}{l}\text { TT } \\
\text { TC } \\
\text { CC }\end{array}$ & $\begin{array}{r}1009.426 \pm 21.102 \\
954.500 \pm 23.148 \\
1015.555 \pm 23.148 \\
\end{array}$ & $\begin{array}{l}2.307 \pm 0.072 \\
2.430 \pm 0.117 \\
2.278 \pm 0.039 \\
\end{array}$ & $\begin{array}{l}1.382 \pm 0.068 \\
1.460 \pm 0.234 \\
1.240 \pm 0.041 \\
\end{array}$ & $\begin{array}{r}56.216 \pm 0.835 \\
55.800 \pm 1.786 \\
57.694 \pm 0.595 \\
\end{array}$ \\
\hline \multirow{4}{*}{$\mathrm{T} 3182 \mathrm{C}$} & DD & $\begin{array}{l}\text { TT } \\
\text { TC } \\
\text { CC }\end{array}$ & $\begin{array}{l}1063.473 \pm 21.992 \\
1121.774 \pm 11.377 \\
1110.470 \pm 23.250\end{array}$ & $\begin{array}{l}2.352 \pm 0.034 \\
2.262 \pm 0.018 \\
2.261 \pm 0.036\end{array}$ & $\begin{array}{l}1.383 \pm 0.033 \\
1.322 \pm 0.017 \\
1.291 \pm 0.034\end{array}$ & $\begin{array}{r}{ }^{a b} 56.968 \pm 0.610 \\
58.507 \pm 0.315 \\
{ }^{b} 58,805 \pm 0.645\end{array}$ \\
\hline & LL & $\begin{array}{l}\text { TT } \\
\text { TC } \\
\text { CC }\end{array}$ & $\begin{array}{r}1003.000 \pm 12.903 \\
981.500 \pm 33.525 \\
956.555 \pm 31.608\end{array}$ & $\begin{array}{l}2.366 \pm 0.018 \\
2.436 \pm 0.047 \\
2.405 \pm 0.044\end{array}$ & $\begin{array}{l}1.519 \pm 0.044 \\
1.578 \pm 0.114 \\
1.578 \pm 0.114\end{array}$ & $\begin{array}{l}56.409 \pm 0.531 \\
53.812 \pm 1.381 \\
56.055 \pm 1.302\end{array}$ \\
\hline & $Y Y$ & $\begin{array}{l}\text { TT } \\
\text { TC } \\
\text { CC }\end{array}$ & $\begin{array}{l}1024.804 \pm 17.266 \\
1020.638 \pm 18.426 \\
1026.166 \pm 45.135\end{array}$ & $\begin{array}{l}2.390 \pm 0.019 \\
2.374 \pm 0.020 \\
2.395 \pm 0.050\end{array}$ & $\begin{array}{l}1.349 \pm 0.035 \\
1.378 \pm 0.037 \\
1.463 \pm 0.091\end{array}$ & $\begin{array}{l}57.800 \pm 0.482 \\
57.555 \pm 0.515 \\
55.783 \pm 1.262\end{array}$ \\
\hline & BB & $\begin{array}{l}\text { TT } \\
\text { TC } \\
\text { CC }\end{array}$ & $\begin{array}{c}991.333 \pm 58.824 \\
1009.500 \pm 25.471 \\
1063.000 \pm 101.887\end{array}$ & $\begin{array}{l}2.353 \pm 0.101 \\
2.280 \pm 0.043 \\
2.330 \pm 0.174 \\
\end{array}$ & $\begin{array}{l}1.343 \pm 0.107 \\
1.258 \pm 0.046 \\
1.070 \pm 0.186 \\
\end{array}$ & $\begin{array}{l}55.466 \pm 1.431 \\
57.956 \pm 0.619 \\
56.400 \pm 2.478 \\
\end{array}$ \\
\hline \multirow{4}{*}{$\mathrm{C} 4858 \mathrm{~A}$} & $\mathrm{DD}$ & $\begin{array}{l}\mathrm{CC} \\
\mathrm{CA} \\
\mathrm{AA}\end{array}$ & $\begin{array}{l}1085.625 \pm 17.124 \\
1121.150 \pm 11.377 \\
1073.000 \pm 68.497\end{array}$ & $\begin{array}{l}2.304 \pm 0.027 \\
2.265 \pm 0.018 \\
2.320 \pm 0.109\end{array}$ & $\begin{array}{l}1.350 \pm 0.025 \\
1.321 \pm 0.017 \\
1.245 \pm 0.103\end{array}$ & $\begin{array}{l}57.771 \pm 0.478 \\
58.460 \pm 0.316 \\
59.900 \pm 0.478\end{array}$ \\
\hline & LL & $\begin{array}{l}\mathrm{CC} \\
\mathrm{CA} \\
\mathrm{AA}\end{array}$ & $\begin{array}{r}956.555 \pm 31.608 \\
981.500 \pm 33.525 \\
1003.000 \pm 12.903\end{array}$ & $\begin{array}{l}2.405 \pm 0.044 \\
2.436 \pm 0.047 \\
2.366 \pm 0.018\end{array}$ & $\begin{array}{l}1.508 \pm 0.108 \\
1.578 \pm 0.114 \\
1.519 \pm 0.044\end{array}$ & $\begin{array}{l}56.055 \pm 1.302 \\
53.812 \pm 1.381 \\
56.409 \pm 0.531\end{array}$ \\
\hline & YY & $\begin{array}{l}\mathrm{CC} \\
\mathrm{CA} \\
\mathrm{AA}\end{array}$ & $\begin{array}{r}{ }^{a} 967.500 \pm 37.304 \\
a_{1041.396 \pm 13.293} \\
b^{b} 964.083 \pm 30.459\end{array}$ & $\begin{array}{l}2.445 \pm 0.042 \\
2.367 \pm 0.015 \\
2.429 \pm 0.034\end{array}$ & $\begin{array}{l}1.301 \pm 0.079 \\
1.384 \pm 0.028 \\
1.341 \pm 0.097\end{array}$ & $\begin{array}{l}58.100 \pm 1.103 \\
57.390 \pm 0.393 \\
58.008 \pm 1.103\end{array}$ \\
\hline & BB & $\begin{array}{l}\mathrm{CC} \\
\mathrm{CA} \\
\mathrm{AA} \\
\end{array}$ & $\begin{array}{r}1009.250 \pm 48.472 \\
1041.111 \pm 32.315 \\
968.857 \pm 36.642 \\
\end{array}$ & $\begin{array}{l}2.347 \pm 0.085 \\
2.242 \pm 0.056 \\
2.328 \pm 0.064 \\
\end{array}$ & $\begin{array}{l}1.275 \pm 0.083 \\
1.167 \pm 0.055 \\
1.375 \pm 0.063 \\
\end{array}$ & $\begin{array}{r}56.657 \pm 0.840 \\
{ }^{a b} 58.966 \pm 0.741 \\
{ }^{\mathrm{b}} 56.657 \pm 1.112 \\
\end{array}$ \\
\hline
\end{tabular}

at $b$, c Values with different superscripts within column are significantly different, ${ }^{*} \mathrm{P}<0.01 ; \mathrm{P}<0.05$

DD: Duroc, LL: Landrace, YY: Yorkshire, BB: Berkshire 
between FABP4 (A1761G) GG subgroup and AG or AA subgroup $(\mathrm{P}<0.05)$. And in Duroc group, it was found that FABP4 (A2521G) AG subgroup showed significantly higher daily gain than AA or GG subgroup, while GG subgroup showed significantly higher feed conversion ratio than AA or AG subgroup. In addition, for FABP4 (T3182C) single nucleotide locus, CC subgroup showed more significant differences once again in lean percentage than TT or TC subgroup. On the other hand, in Landrace group, it was found that FABP4 (T2713C) TT subgroup showed significantly high involvement in feed conversion ratio and backfat thickness. And FABP'4 TC or CC subgroup showed much less significances than TA subgroup $(\mathrm{P}<0.02)$. Also, Yorkshire group showed higher significances in daily gain en bloc than other breed groups. Here, FABP4 (A1761G) $A G$ or $G G$ subgroup showed more significant differences than AA subgroup. (A2521G) locus, (T2713C) locus and (C4858A) locus of Yorkshire group showed more significant differences in daily gain than those of other groups $(\mathrm{P}<0.05)$. In Berkshire group, it was found that FABP4 (C4858A) CA subgroup showed more significant differences in backfat thickness and lean percentage than CC or AA subgroup. As a result, it was found that there were more or less significant differences in daily gain, feed conversion ratio, backfat thickness and lean percentage among genotype subgroups in 4 breeds, which get involved in respective single nucleotides of FABP4. Therefore, FABP4 gene might be associated with increasing body fat which involves high level of daily gain, backfat thickness and lean percentage depending on genotypes. In addition, feed conversion ratio calculated now in KSA Laboratory is determined as total amount of feed taken by 2 head of black swine until they gain weight as heavy as $180 \mathrm{~kg}$. That is why it fails to correctly reflect differences in feed conversion ratio depending on FABP4 genotype of each subgroup. According to the results of this study, it was found that different genotype subgroups showed excellent level of daily gain, backfat thickness and lean percentage depending on FABP4 SNP genotype and there are associations among high daily gain, high body fat and high lean percentage in subgroups of different FABP4 genotypes.

Hence, the results of this study demonstrated that FABP4 genotypes have significant effects on daily gain, backfat thickness, feed conversion ratio and lean percentage which are all measured in KSA Laboratory. So if they are applied to screening index equation to measure the performances of black swine, FABP4 genotypes will be useful in accurate identification of superior swine. Moreover, there are significant differences in frequency of FABP4 genotype depending on swine breeds, so it will be helpful to use FABP4 genotypes for each breed in early screening superior swine.

\section{Acknowledgements}

We are deeply grateful to National Institute of Animal Science (NIAS) for financial supports in our ordinary research expenses. In addition, we give great acknowledgement to parties concerned with financial supports in research budget for a Bio Green 21 Project titled "the Development of DNA Chip and Industrial System for Better Flesh Quality Based on the Genetic Information of Swine" (20050401-034-804) in Korea.

\section{References}

1. Andersson, L., G. S. Haley, H. Ellegren, S. A. Knott, M. Johansson. 1994. Genetic mapping of quantitative trait 1oci for growth and fatness in pigs. Science 263, 1771-1174.

2. Berg, F., S. Stern, K. Andersson, L. Andersson and M. Moller. 2006. Refined localization of the FAT1 quantitative trait locus on pig chromosome 4 by marker-assisted backcrossing. BMC Genetics 7, 7-17.

3. De Koning, D. J., B. Harlizius, A. P. Rattink, M. A. Groenen, E. W. Brascamp and J. A. van Arendonk. 2001. Detection and characterization of quantitative trait loci for growth and reproduction traits in pig. J. Anim. Sci. 79, 2812-2819.

4. Gerbens, F., A. Jansen, A. J. van, Erp, F. Harders, T. H. Meuwissen, G. Rettenberger, J. H. Veerkamp and M. F. te Pas. 1998. The adipocyte fatty acid-binding protein locus: Characterization and association with intramuscular fat content in pig. Mamm. Genome 9, 1022-1026.

5. Haunerland, N. H. and F. Spener. 2004. Fatty acid-binding protein-insights from genetic manipulation. Prog. Lipid Res. 43, 328-349.

6. Mercade, A., M. Perez-Enciso, L. Varona, E. Alves, L. J. Noguera, A. Sanchez and M. J. Folch. 2006. Adipocyte fatty - acid binding protein is closely associated to the porcine FAT1 locus on chromosome 4. J. Anim. Sci. 84, 2907-2913.

7. Milan, D., J. P. Bidanel, N. Lannuccelli, J. Riquet, Y. Amigues, J. Gruand, P. le Roy, C. Renard and C. Chevalet. 2002. Detection of quantitative trait loci for carcass composition trait in pig. Genet. Sel. Evol. 34, 705-728.

8. Ojeda, A., J. Rozas, J. M. Folch and M. Perez-Enciso. 2006. 
Unexpected high polymorphism at the FABP4 gene unveils a complex history for pig population. Genetics 174, 2119-2127.

9. Paszek, A. A., P. J. Wilkie, G. H. Flickinger, L. J. Alexander, C. W. Beattie and L. B. Schook. 1999. Interval mapping of growth in divergent swine cross. Mamm. Genome 10, 117-122.
10. Van Laere, A. S., M. Nguyen, M. Braunschweig, C. Nezer, C. Collette, L. Moreau, A. L. Archibald, C. S. Haley, N. Buys, M. Tally, G. Andersson, M. Georges and L. Andersson. 2003. Aregulatory mutation in IGF2 causes a major QTL effect on muscle growth in the pig. Nature 23, 832-836.

\section{초록 : FABP4 유전자의 단일염기 다형성에 관한 연구}

김상욱 $\cdot$ 정지혜 $\cdot$ 김관석 ${ }^{*}$ 이철구 ${ }^{2} \cdot$ 김종주 $^{3} \cdot$ 최봉환 $^{4} \cdot$ 김태헌 $^{4} \cdot$ 송기덕 $^{5} \cdot$ 조병욱 ${ }^{*}$

(부산대학교 생명자원과학대학 생명자원과학부, ${ }^{1}$ 충북대학교 축산학과, ${ }^{2}$ 고려대학교 생명공학부,

${ }^{3}$ 영남대학교 생명공학부, ${ }^{4}$ 농촌진홍청 축산연구소, ${ }^{5}$ 미국국립보건원)

본 연구는 돼지 4번 염색체에서 FAT1 좌위의 후보유전자인 Adipocyte Fatty-Acid 결합단백질 (FABP4) 유전자 에서 8개의 Single Nucleotide Polymorphisms (SNPs) 를 발견하였다. Duroc, Landrace, Berkshire, Yorkshire를 기초 축으로 이용한 800 두에 대해 FABP4 유전자의 단일염기 분석과 PCR-RFLP를 이용하여 그 다형성을 조사하 고 돼지의 일당증체량, 등지방두께, 사료요구율, 정육율과 그 유전자형 간의 연관성을 규명하고자 실시하였다. FABP4 유전자에 대해 각 단일염기에 관한 PCR-RFLP롤 이용하여 400 800 bp 산물을 증폭한후 각각의 제한효소 로 사용하여, 얻어진 FABP4 유전자의 빈도는 품종별로 다르게 나타넜다. 통계적 분석을 통하여 각 유전자형에 대한 경제형질과 연관성을 분석한 결과 일당증체량, 등지방두께, 정육율, 사료요구량은 다른 유전자형을 가진 개 체들이 유의적으로 우수한 능력을 보였다 ( $\mathrm{P}<0.05)$. FABP4유전자는 일당증체량, 정육율, 등지방두께 에 높은 연 관성이 있음을 관찰하였다. 따라서 돼지의 성장과 정육율에 관련된 선발력을 높이기 위해서 FABP4 유전자의 다 형성 분석에서 검증된 PCR marker를 우량돼지육종 계획에 있어 분자생물학적 선발 marker로 사용할 수 있을 것으로 사료된다. 